\title{
Influencia de las disposiciones en el desarrollo del pensamiento crítico y el aprendizaje de las Ciencias Naturales
}

\author{
Influence of the provisions in the \\ development of critical thinking and \\ Learning Science
}

\section{Influência das disposições no desenvolvimento do pensamento crítico e a aprendizagem das Ciências Naturais}

Nidia Yaneth Torres Merchán ${ }^{1}$

\begin{abstract}
RESUMEN
El trabajo que se presenta a continuación manifiesta la importancia de considerar en el proceso de aprendizaje las disposiciones y las actitudes de los estudiantes; para lo cual se realizó una caracterización de estas en un grupo de estudiantes de educación media, utilizando un cuestionario que hace parte deltest HCTAES, propuesto por Halpern (1998). Este instrumento permite evidenciar la motivación de los estudiantes en clases de ciencias y su capacidad de pensamiento crítico, por lo que en gran medida hace que los estudiantes toman conciencia de lo que aprenden durante su formación. Palabras-clave: disposiciones; pensamiento crítico; enseñanza de las ciencias.
\end{abstract}

\section{ABSTRACT}

The work presented here shows the importance of considering in the process of learning the rules and attitudes of students, for whom we made a

${ }^{1}$ Docente de la Facultad de Ciencias de la Educación, Universidad Pedagógica y Tecnológica de Colombia. Licenciada en Biología y Química, Universidad Pedagógica y Tecnológica de Colombia. Magister en Docencia de La Química, Universidad Pedagógica Nacional, Bogotá, Colombia. 
characterization of such aspects in a group of middle school students using a questionnaire that is part of the HCTAES test proposed by Halpern (1998). This instrument can reveal the motivation of students in science classes and critical thinking skills, so the students greatly have become aware of what they learn during training.

Keywords: provisions; critical thinking; science education.

\section{RESUMO}

O trabalho apresentado aqui mostra a importância de se considerar no processo de aprendizagem as disposições e as atitudes dos alunos, para os quais fizemos uma caracterização destes em um grupo de estudantes do ensino médio através de um questionário que faz parte do HCTAES, teste proposto por Halpern (1998). Este instrumento pode revelar a motivação dos alunos nas aulas de ciências e habilidades de pensamento crítico, pois em grande medida faz com que os alunos tomem conhecimento do que eles aprendem durante a sua formação.

Palavras-chave: disposições; pensamento crítico; educação científica.

\section{Introducción}

Actualmente es indispensable fomentar el interés de los estudiantes para involucrarse de manera activa y responsable en su propio aprendizaje, hay acuerdo en sostener que una de las causas de la falta de interés reside en una actitud negativa de los estudiantes hacia el aprendizaje, en este caso el aprendizaje de las ciencias (VÁZQUEZ; MANASSERO, 2008).Dado que el principal interés considerado por algunos docentes en la enseñanza de las ciencias son los contenidos, se debe considerar acciones tendientes a promover en los alumnos actitudes favorables hacia el aprendizaje de las ciencias y el desarrollo del pensamiento crítico.

El objetivo de este trabajo fue observar de que manera influyen las disposiciones y las actitudes en los estudiantes de educación media en el aprendizaje de las ciencias, por lo que en este trabajo se considera importante recurrir a la visión pragmática dada por la Teoría Uno de Perkins (2003), que se sintetiza como: "La gente aprende más cuando tiene una oportunidad razonable y una motivación para hacerlo". Por los que esta teoría es una concepción acerca de la buena enseñanza basada en el sentido común. No hace referencia a ninguna 
teoría educativa específica sino que hace insistencia en que toda práctica docente debe atender a garantizar los siguientes aspectos:

$>$ Información clara: Descripción y ejemplos de objetivos, conocimientos requeridos y resultados esperados;

$>$ Práctica Reflexiva: Oportunidad para el alumno de ocuparse activa y reflexivamente de aquello que deba aprender;

$>$ Realimentación informativa: Consejos claros y precisos para que el alumno mejore su rendimiento y pueda proceder de manera más eficaz;

$>$ Fuerte motivación intrínseca y extrínseca: Actividades ampliamente recompensadas, sea porque son muy interesantes y atractivas en sí mismas o porque permiten obtener otros logros que importan al alumno.

Perkins, Jay y Tishman (1993) consideran tener en cuenta los siguientes componentes psicológicos para desarrollar disposiciones como son: sensibilidad como la percepción de un comportamiento particular apropiado. Inclinación, el ímpetu sentido hacia un comportamiento, y habilidad, la capacidad básica de llevar a cabo el comportamiento. Siegel (1990) manifiesta que esta tendencia está compuesta por objetividad, honestidad intelectual, imparcialidad, el deseo de hacer concordar el juicio y la acción con el principio y un compromiso para buscar y evaluar razones. Costa (1991) no utiliza el término "disposiciones de pensamiento", sino hace referencia a "pasiones de la mente". Identifica cinco pasiones claves que caracterizan al buen pensador: eficacia, flexibilidad, conciencia, destreza e interdependencia.

\section{Influencia de las disposiciones en el pensamiento crítico}

Hasta 1994 se utilizaron Test para evaluar solo habilidades en pensamiento crítico y trabajos realizados por Watson y Glaser (1984), Ennis y Weir (1985), Ennis y Millman (1985a, 1985b) inferían que si se tenían buenas habilidades entonces se poseían buenas disposiciones, hasta que en un primer estudio llevado a cabo por Giancarlo y Facione (1994), con una muestra de 193 estudiantes de secundaria, se encontró una correlación positiva $(\mathrm{r}=0.41)$ entre las puntuaciones totales del Test de Habilidades de Pensamiento Crítico de California (CCTST) y las del Inventario de Disposiciones del Pensamiento Crítico de California (CCTDI) (FACIONE; FACIONE, 1992), esta relación fue significativa $(\mathrm{p}<.05)$. En posteriores estudios con un mayor número de estudiantes los mismos autores encontraran que quien tiene niveles altos de habilidades también los tiene de disposiciones y viceversa; estos estudios de disposiciones evalúan 
exclusivamente las disposiciones entendidas como actitudes intelectuales; así, evalúan disposiciones como la sistematicidad, el análisis, la imparcialidad, la curiosidad, la búsqueda de la verdad y la confianza en la razón, pero no evalúan la motivación para emplear el pensamiento crítico; Halpern (2006) ha desarrollado un Test para contribuir a esta información: ha creado un instrumento para su valoración "HCTAES - Test de Halpern para la Evaluación del Pensamiento Crítico mediante Situaciones Cotidianas". Estudios de Saiz (2006) en la Universidad de Salamanca reflejan que existe una relación positiva entre la motivación general para usar el pensamiento crítico y la destreza en su uso.

La concepción básica detrás de las disposiciones es algo así: una gran parte de ser inteligente significa ser capaz de pensar bien; y la gente que piensa bien tiene disposiciones de pensamiento sólidas. Por consiguiente una gran parte de ser inteligente significa tener disposiciones de pensamiento sólidas. Esto no implica que todos los buenos pensadores tengan las mismas disposiciones de pensamiento, o que todos los buenos pensadores tengan disposiciones de pensamiento igualmente sólidas (SALOMON, 1994).

\section{Metodología}

Se utiliza un cuestionario con 38 afirmaciones, que hacen parte del test HCTAES y que pretende evaluar la disposición de los aspectos del pensamiento crítico, por los se proporcionan cuestionarios a los participantes. El instrumento denominado: "Escala de conciencia" (COSTA; MCCRAE, 1992) es una de las evaluaciones que se utiliza con el Factor Teoría de la Personalidad, los estudiantes utilizan siete elementos que les permite hacer una descripción de su personalidad; de la misma manera el cuestionario está diseñado para evaluar una tendencia del individuo a "participar y a disfrutar de sus esfuerzos cognitivos" (CACIOPPO; PETTY; FEINSTEIN; JARVIS, 1996) por los que cada escala tiene un significado de la siguiente manera:

$1=$ Extremadamente imprecisa.

$2=$ Moderadamente imprecisa.

$3=$ Ligeramente imprecisa.

$4=$ Ni precisa ni imprecisa.

$5=$ Ligeramente precisa

$6=$ Moderadamente precisa.

$7=$ Extremadamente precisa.

Para lo cual cada estudiante elige un número para cada afirmación (Anexo 1). 
En este trabajo participaron 30 estudiantes que cursan la educación media de un colegio de la Ciudad de Tunja, Boyacá, con edades entre los 15 y los 16 años. La prueba fue resulta de manera individual por un tiempo de 20 minutos.

Seguidamente, se realizó una entrevista que permitiera manifestar a los estudiantes de manera espontanea el concepto de disposición, su importancia en el proceso de formación y su relación con el aprendizaje de las Ciencias Naturales.

\section{Resultados y discusión}

La disposición es una dimensión del pensamiento crítico que no es sólo si la gente puede pensar sino si se sienten inclinados a hacerlo. Por lo que los resultados encontrados en este cuestionario fueron analizados desde dos categorías: la escala de conciencia y el esfuerzo cognitivo.

\section{Escala de conciencia}

En esta categoría básicamente se detallan aspectos sobre organización, planeación de actividades y utilización adecuada del tiempo. Aunque la mayoría de los estudiantes reconoce la importancia de dichos aspectos, no se involucran a profundidad en actividades que les permitiría realizar mejor sus actividades tales como: dejar las cosas tiradas por ahí, no culminar las actividades propuestas, hacer las cosas a medias.

La Figura 1 manifiesta que el $28 \%$ de los estudiantes participantes manifiestan actitudes positivas frente a la organización y planeación de sus actividades. Estas incluyen una actitud positiva hacia situaciones ambiguas y complejas, una preferencia hacia lo nuevo e incongruente, y la intención de buscar este tipo de situaciones, o inclusive moldear situaciones de forma tal que concuerden con la preferencia (SOLOMON, 1994).

\section{Esfuerzo cognitivo}

Esta categoría involucra aquellos aspectos relacionados con la preferencia o no por desarrollar situaciones complejas y en las que los estudiantes deban 


\section{Escala de Conciencia}

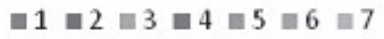

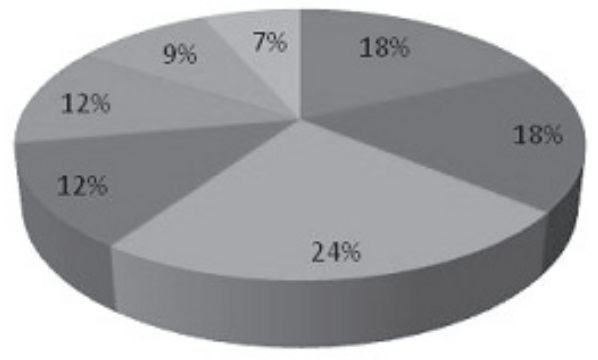

FIGURA 1 - PORCENTAJE DE ESCALA DE CONCIENCIA MANIFESTADA POR LOS ESTUDIANTES

hacer un análisis profundo de las situaciones problémicas y que además disfruten y reflexionen críticamente la solución de estas situaciones.

Los resultados arrojados por esta prueba permiten evidenciar que los estudiantes manifiestan actitudes negativas frente a su esfuerzo cognitivo, por los que prefieren aquellas situaciones en que su solución sea de manera rápida y no implique profundización de muchos aspectos. Por lo que solo el $24 \%$ de la población manifiesta actitudes positivas frente al agrado de situaciones complejas que requieran más tiempo para pensar, tal como es señalado en la Figura 2.

De acuerdo con Halpern (2006), ser un buen pensador significa no solo poseer habilidades de pensamiento crítico sino también poseer motivaciones, actitudes, valores y hábitos mentales; para esto se debe partir de los intereses propios de los estudiantes, de sus necesidades cognitivas y de las temáticas disciplinares con repercusiones sociales; transformando la visión que se tiene de una enseñanza tradicional por una enseñanza de las ciencias que permita no solo comprender los conceptos disciplinares, sino también favorecer procesos de pensamiento crítico.

Como segundo momento de este trabajo se realizó una entrevista en la que se evidenciaba la importancia de considerar las disposiciones de los estudiantes y como estas influyen en el aprendizaje de las Ciencias Naturales.

Los resultados de la entrevista manifiestan que los estudiantes conciben las disposiciones de la siguiente manera: 


\section{Esfuerzo Cognitivo}

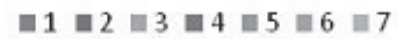

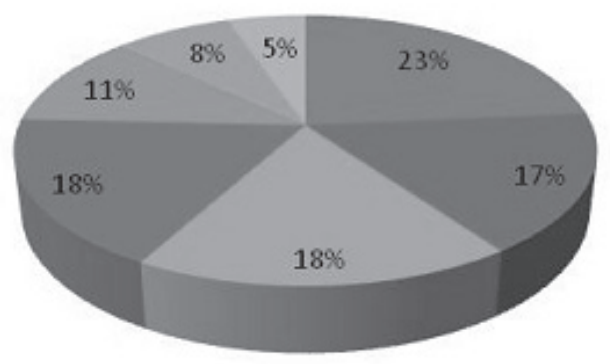

FIGURA 2 - PORCENTAJE DE ESCALA DE ESFUERZO COGNITIVO CONSIDERADO POR LOS ESTUDIANTES

E1. Aquello para estar bien la clase.

E2. Lo que me permite aprender mejor.

E3. Es una preferencia a estar en unas clases y en otras no.

E4. Es la motivación que tenemos para aprender, pero que depende de la clase, por ejemplo el tema, si el tema se comprende o no.

De acuerdo con Ennis (1996), la disposición de pensamiento se define como una tendencia para hacer algo en determinadas condiciones y estar propenso o susceptible a un estado particular, o experimentar un cambio particular.

De la misma manera se les cuestionó acerca de lo que entendían por disposiciones negativas a los cual respondieron lo siguiente:

E3. Lo que no deja hacer bien las tareas.

E5. Es todo lo malo que no permite aprender.

E6. Es la pereza.

E7. Es la falta de organización y planeación en el estudio.

Considerando las respuestas de los estudiantes es necesario proporcionar espacios donde los estudiantes hagan una reflexión consciente, crítica y respon- 
sable acerca de sus actitudes y la influencia dentro de su proceso de formación, es así como las disposiciones positivas permiten mantener una mente abierta, flexible, justa cuando se trata de evaluar, que construye conocimientos, clara en respecto a los problemas o las situaciones que requieren la emisión de un juicio; ordenada cuando se enfrenta a situaciones de mayor complejidad; diligente en la búsqueda de información relevante; razonable en la selección de criterios; enfocada en preguntar, indagar, investigar; persistente en la búsqueda de resultados tan precisos como las circunstancias y el problema o la situación lo permitan; mientras que las disposiciones negativas permiten mantener un pensamiento unilateral, cerrado, aburrido y con muy poca motivación por aprender cosas nuevas (FACIONE; FACIONE, 1992).

Se les cuestiono ¿Cuales son las disposiciones que tienen frente al aprendizaje de las ciencias?

La mayoría de los estudiantes manifestó disposiciones negativas frente al aprendizaje de las ciencias en afirmaciones como las siguientes:

E1. Las ciencias como la fisica y química son dificiles, porque estas utilizan números, me va mejor en Biología, no soy bueno para los números.

E2. Porque los docentes siempre explican los ejercicios fáciles y nos dejan los dificiles.

E3. Porque entiendo en el momento de la explicación pero en la evaluación se me olvida.

E4. Porque siempre se utilizan ejercicios que en muchos de los casos no se vuelven a utilizar nunca.

E5. Porque hay muchas tareas y a veces no se revisan.

Las afirmaciones anteriores permiten inferir que se hace necesario desarrollar estrategias que promuevan disposiciones positivas hacia el aprendizaje de las ciencias y que originen el desarrollo del pensamiento crítico, para que el aprendizaje de las ciencias no se torne en aburrimiento y experiencias de fracaso escolar (MURPHY; BEGGS, 2003). Por lo que también se hace necesario incluir actividades relacionadas con actividades de la vida cotidiana y que aborden aspectos interdisciplinarios, donde los estudiantes vean la utilidad de lo que aprenden, de la misma manera se deben realizar sesiones de retroalimentación en cada actividad para detectar las falencias y evaluar el progreso que presentaron los estudiantes a nivel disciplinar, actitudinal, afectivo, emotivo y de desarrollo de pensamiento crítico; de forma tal que se planearon mejores estrategias para superar las debilidades y facilitar su proceso de aprendizaje. 
De igual forma, los estudiantes manifestaron la importancia y atracción hacia el trabajo colectivo ya que les permite la comprensión de diversos conceptos, además de generar un ambiente participativo entre todos los miembros de grupo, por lo cual los docentes debemos preocuparnos por diseñar actividades que permitan generar curiosidad y motivar al aprendizaje.

\section{Conclusiones}

Las disposiciones contribuyen al desarrollo general de pensamiento, estas son evidenciadas por los estudiantes en el desarrollo de sus prácticas de aula y prácticas contextuales, por lo que los educadores deben proporcionar espacios y actividades que les permitan desarrollar disposiciones positivas en su proceso de aprendizaje.

La mayoría de los estudiantes en este estudio concibe la importancia de las disposiciones positivas pero no desarrolla actividades que reflejen su utilidad, por lo que se deben considerar espacios que les permitan reflexionar a los estudiantes acerca de su propio proceso de aprendizaje y de las disposiciones, actitudes y valores que permiten desarrollar un pensamiento crítico para la toma de solución de problemas y la toma de decisiones responsablemente.

Los docentes deben ayudar a sus estudiantes a desarrollar disposiciones de pensamiento sólidas y estables, con la estructuración de experiencias donde los estudiantes elaboran razonamientos por si mismos que les permitan una constante interacción son su familia, compañeros y docentes.

\section{REFERENCIAS}

CACIOPPO, J. T.; PETTY, R. E.; FEINSTEIN, J.; JARVIS, W. B. G. Dispositional differences in cognitive motivation: The life and times of individuals varying in need for cognition. Psychological Bulletin, n. 119, p. 197-253, 1996.

COSTA, A. L. Developing Minds: A resource book for teaching thinking. USA: ASCD, 1991. v. 1.

COSTA, P.; MCCRAE, R. The NEO Five-Factor Inventory (NEO-FFI). [Spanish translation: Inventario de personalidad NEO revisado (NEO PI-R). Inventario NEO 
reducido de cinco factores (NEO-FFI). Madrid: TEA, 1999]. Odessa, FLA: PAR Psychological Assessment Resources, 1992.

ENNIS, R. Critical thinking. Upper Saddle River, New York: Prentice-Hall, 1996.

ENNIS, R.; MILLMAN, J. Cornell Critical Thinking Test, level X. Pacific Grove, CA: Critical Thinking Books and Software, 1985a.

. Cornell Critical Thinking Test, level Z. Pacific Grove, CA: Critical Thinking Books and Software, $1985 b$.

ENNIS, R.; WEIR, E. The Ennis-Weir Critical Thinking Essay Test. Pacific Grove, CA: Critical thinking Books and Software, 1985.

FACIONE, P.; FACIONE, N. The California Critical Thinking Dispositions Inventory (CCTDI); and the CCTDI Test manual. Millbrae, CA: The California Academic Press, 1992.

GIANCARLO, C.; FACIONE, N. A study of the critical thinking disposition and skill of spanish and English speaking students at Camelback high school. Millbrae, CA: The California Academic Press, 1994.

HALPERN, D. Teaching critical thinking for transfer across domains. American Psychologist, v. 53, n. 4, p. 449-455, 1998.

. Halpern Critical Thinking Assessment Using Everyday Situations: Background and scoring standards ( $2^{\circ}$ Report). Unpublished manuscript. Claremont, CA: Claremont McKenna College, 2006.

MURPHY, C.; BEGGS, J. Children perceptions of school science. School Science Review, v. 84, n. 308, p. 109-116, 2003.

PERKINS, D. La escuela Inteligente. Barcelona: Editorial GEDISA S.A., 2003. Capítulo 3.

PERKINS, D.; JAY, E.; TISHMAN, S. Beyond abilities: A dispositional theory of thinking. The Merrill-Palmer Quarterly, v. 39, n. 1, p. 1-21, 1993.

SAIZ, C. Pensamiento crítico. 2006. Disponible en: $<$ http://www.pensamiento-crítico. com/halpernrealizar1006.htm>. Acceso en: 5/5/2008.

SIEGEL, H. The justification of critical thinking as an educational ideal. In: Education reason: rationality, critical thinking and education. New York: Routledge, 1990.

SOLOMON, J. The rise and fall of constructivism. Studies in Science Education, n. 23, p. 1-19, 1994. 
VÁZQUEZ, A.; MANASSERO, M. A. El Declive de las Actitudes Hacia la Ciencia de los Estudiantes: Un Indicador Inquietante para la Educación Científica. Rev. Eureka Enseñ. Divul. Cien., v. 5, n. 3, p. 274-292, 2008.

WATSON, G.; GLASER, E. M. The Watson-Glaser Critical Thinking Appraisal. San Antonio, TX: The Psychological Corporation, 1984.

Texto recebido em 23 de fevereiro de 2011.

Texto aprovado em 30 de setembro de 2011.

\section{ANEXO 1}

TEST PARA EVALUAR DISPOSICIONES DE PENSAMIENTO

\section{Por favor, valora cada una de las siguientes afirmaciones en función de lo precisas que sean para describirte. Utiliza una escala de 7 puntos donde:}

1 = Extremadamente imprecisa. 2 = Moderadamente imprecisa. 3 = Ligeramente imprecisa. $4=$ Ni precisa ni imprecisa. $5=$ Ligeramente precisa. $6=$ Moderadamente precisa. $7=$ Extremadamente precisa. Elige un número para cada afirmación.

\begin{tabular}{|l|c|}
\hline & 1234567 \\
\hline 1) Estoy siempre preparado. & O O O O O O O \\
\hline 2) Dedico mucha atención a los detalles. & O O O O O O O \\
\hline 3) Realizo mis quehaceres enseguida. & O O O O O O O \\
\hline 4) Me gusta el orden. & O O O O O O O \\
\hline 5) Sigo el plan que me trazado. & O O O O O O O \\
\hline 6) Soy preciso en mi trabajo. & O O O O O O O \\
\hline 7) Actúo de acuerdo con un plan. & O O O O O O O \\
\hline
\end{tabular}




\begin{tabular}{|c|c|}
\hline & 1234 \\
\hline 8) No paro hasta que todo está perfecto. & OOOOOOO \\
\hline 9) Hago planes y los cumplo. & $\mathrm{OOOOOOO}$ \\
\hline 10) Me encanta el orden y la regularidad. & $\mathrm{OOOOOOO}$ \\
\hline 11) Me gusta la limpieza. & OOOOOOO \\
\hline 12) Dejo las cosas tiradas por ahí. & $\mathrm{OOOOOOO}$ \\
\hline 13) Hago las cosas mal. & $\mathrm{OOOOOOO}$ \\
\hline $\begin{array}{l}\text { 14) Con frecuencia me olvido de dejar las cosas } \\
\text { en su sitio. }\end{array}$ & $\mathrm{OOOOOOO}$ \\
\hline 15) Evito mis obligaciones. & $\mathrm{OOOOOOO}$ \\
\hline 16) Descuido mis obligaciones. & $\mathrm{OOOOOOO}$ \\
\hline 17) Malgasto mi tiempo. & $\mathrm{OOOOOOO}$ \\
\hline 18) Hago las cosas a medias. & $\mathrm{OOOOOOO}$ \\
\hline 19) Me cuesta ponerme a trabajar en serio. & $\mathrm{OOOOOOO}$ \\
\hline 20) No limpio mi habitación. & $\mathrm{OOOOOOO}$ \\
\hline $\begin{array}{l}\text { 21) Prefiero los problemas complicados a los } \\
\text { sencillos. }\end{array}$ & OOOOOOO \\
\hline $\begin{array}{l}\text { 22) Me gusta tener la responsabilidad de manejar } \\
\text { una situación que exige pensar mucho. }\end{array}$ & $\mathrm{OOOOOOO}$ \\
\hline 23) Pensar no es divertido para mí. & OOOOOOO \\
\hline $\begin{array}{l}\text { 24) Prefiero hacer cosas que requieran pensar poco } \\
\text { a otras que signifiquen un reto para mis capacidades } \\
\text { intelectuales. }\end{array}$ & OOOOOOO \\
\hline $\begin{array}{l}\text { 25) Intento anticipar y evitar situaciones en las que } \\
\text { es muy probable que tenga que pensar en profun- } \\
\text { didad sobre algo. }\end{array}$ & $\mathrm{OOOOOOO}$ \\
\hline $\begin{array}{l}\text { 26) Me gusta pensar profundamente y durante } \\
\text { horas. }\end{array}$ & $\mathrm{OOOOOOO}$ \\
\hline $\begin{array}{l}\text { 27) Sólo pienso con esfuerzo en la medida en que } \\
\text { lo necesito. }\end{array}$ & OOOOOOO \\
\hline $\begin{array}{l}\text { 28) Prefiero pensar en proyectos del día a día, que } \\
\text { en otros a más largo plazo. }\end{array}$ & OOOOOOO \\
\hline $\begin{array}{l}\text { 29) Me gustan las tareas que, una vez que las do- } \\
\text { mine, exijan pensar poco. }\end{array}$ & OOOOOOO \\
\hline
\end{tabular}




\begin{tabular}{|l|c|}
\hline \multicolumn{2}{|l|}{} \\
\hline $\begin{array}{l}\text { 30) La idea de confiar en el pensamiento para llegar } \\
\text { a la cima me gusta. }\end{array}$ & O O O O O O O \\
\hline $\begin{array}{l}\text { 31) Realmente disfruto con tareas que supongan } \\
\text { ofrecer soluciones nuevas a los problemas. }\end{array}$ & O O O O O O O \\
\hline $\begin{array}{l}\text { 32) Aprender nuevas formas de pensar no es nada } \\
\text { interesante para mí. }\end{array}$ & O O O O O O O \\
\hline $\begin{array}{l}\text { 33) Prefiero que mi vida esté llena de problemas } \\
\text { que deba resolver mediante el ingenio. }\end{array}$ & O O O O O O O \\
\hline $\begin{array}{l}\text { 34) La noción de pensamiento abstracto es atractiva } \\
\text { para mí. }\end{array}$ & O O O O O O O \\
\hline $\begin{array}{l}\text { 35) Prefiero una tarea que sea intelectual, difícil } \\
\text { e importante, a otra algo importante, pero que no } \\
\text { exija pensar mucho. }\end{array}$ & O O O O O O O \\
\hline $\begin{array}{l}\text { 36) Me siento más aliviado que satisfecho cuando } \\
\text { termino una tarea que exija mucho esfuerzo mental. }\end{array}$ & O O O O O O O \\
\hline $\begin{array}{l}\text { 37) Es suficiente para mí que una cosa funcione; } \\
\text { no me preocupa el cómo o el por qué de su fun- } \\
\text { cionamiento. }\end{array}$ & O O O O O O O \\
\hline $\begin{array}{l}\text { 38) Normalmente, termino reflexionando sobre } \\
\text { las cuestiones aún cuando no me afecten perso- } \\
\text { nalmente. }\end{array}$ & O O O O O O O \\
\hline
\end{tabular}

FIN DE LA PRUEBA. 